\title{
Medical Images Classification and Diagnostics Using Fuzzy Neural Networks
}

\author{
Yuriy Zaychenko ${ }^{1}$, Aghaei Agh Ghamish Ovi Nafas ${ }^{2}$ \\ ${ }^{1}$ Institute for Applied System Analysis, Igor Sikorsky Kiev Polytechnic Institute, Kiev, Ukraine \\ ${ }^{2}$ Department of Applied Mathematics, Igor Sikorsky Kiev Polytechnic Institute, Kiev, Ukraine \\ Email address: \\ zaychenkoyuri@ukr.net (Y.Zaychenko), ovinafas@yahoo.com (A. A. G. O. Nafas)
}

\section{To cite this article:}

Yuriy Zaychenko, Aghaei Agh Ghamish Ovi Nafas. Medical Images Classification and Diagnostics Using Fuzzy Neural Networks. American Journal of Neural Networks and Applications. Vol. 5, No. 2, 2019, pp. 45-50. doi: 10.11648/j.ajnna.20190502.11

Received: July 23, 2019; Accepted: August 19, 2019; Published: September 9, 2019

\begin{abstract}
The problem of medical images of cervix epithelium classification for express diagnostics is considered...The following states of cervix epithelium are to be recognized and classified: normal state - columnar epithelium; squamous epithelium (normal state); metaplasia-benign changes of cervix uterus epithelium; CIN1-displasia of light degree, CIN 2-displasia of middle degree, CIN 3-displasia of high degree- intra-epithelium cancer: For its solution the application of fuzzy neural network (FNN NEFClass M) is suggested. The application of FNN is grounded by its following properties: it may work with fuzzy and qualitative information; it has accelerated convergence as compared with crisp classification methods; it enables to attain better classification accuracy than conventional classifiers. The structure of FNN NEFClass and its model description are presented. Training algorithm stochastic gradient descent for membership functions of fuzzy sets is considered and implemented. Data set of medical images of cervix epithelium which was obtained by special device colposcope is described and some images are presented. The experimental investigations of FNN NEFClass application for medical images recognition on real data are carried out, the results are presented. The comparison with NN Back Propagation, RBF NN and cascade RBF NN was made and estimation of efficiency of the suggested approach was performed. The problem of reduction of features number in classification tasks using principal component method (PCM) method is considered and implemented.
\end{abstract}

Keywords: Medical Images Classification, Medical Diagnostics, FNN NEFClass, Training, Cascade RBFNN, Features Selection, PCM

\section{Introduction}

An important application sphere of information technologies is the problem of classification of optical medical images and diagnostics in medicine. The advantages of medical diagnostics systems are speed, automation and stability of work which make them very comfortable tools for express medical diagnostics. Despite young age of medical informatics which doesn't exceed 30 years information technologies in a whole are fast penetrating in various spheres of medicine and health defense (family medicine, insurance medicine, integration in European medical space, etc.).

The latest achievements in images processing technologies and machine learning enable to construct systems of automatic detection and diagnostics that may help pathologists-anatomists to make true diagnosis and accelerate his work. Traditional methods of medical images recognition are based on textual descriptors. They use texture features that are most of which are problem-specific and have shown to be complementary in medical images. But they lack generalization ability and demand to construct new textual descriptors for another image [1]. Therefore deep learning and convolutional neural networks $(\mathrm{CNN})$ became powerful and efficient tools for processing and pattern recognition of medical images. Nowadays $\mathrm{CNN}$ are widely used for medical images of human organs classification obtained by different sources (MRT, CT, X -rays images, etc.). The main advantages of $\mathrm{CNN}$ are abilities to learn presentations, extract informative features of images and application of efficient training methods for end-to- end learning [2, 3]. Therefore $\mathrm{CNN}$ now are widely used for medical images analysis and classification, especially for cancer diagnostics. Usually CNN consists of convolutional layers, pooling layers which are used or features extraction and decision layer-MLP (multilayer 
perceptron) as classification unit [2, 3]. An interesting approach based on application of dual deep convolutional neural network with a synergic signal system is suggested in the study [4]. The synergic signal is used to verify whether the input image pair belongs to the same category and to give the corrective feedback if a synergic error exists. Synergic deep learning (SDL) model can be trained 'end to end'. In the test phase, the class label of an input can be predicted by averaging the likelihood probabilities obtained by two convolutional neural network components. Up to date a lot of modern methods for medical images classification were developed. A comprehensive review of modern classification techniques is presented in book [5]. The book covers several complex image classification problems using pattern recognition methods, including Artificial Neural Networks (ANN), Support Vector Machines (SVM), Bayesian Networks (BN) and deep learning. Further, numerous data mining techniques are discussed, as they have proven to be good classifiers for medical images. To the problem of recognition time-dependent data sets of medical images is devoted the monograph [6]. An important feature of this book is the exploration of different approaches to handle and identify time dependent biomedical images. Biomedical imaging analysis and processing techniques deal with the interaction between all forms of radiation and biological cells or tissues in order to visualize small particles and opaque objects and to enable efficient recognition of biomedical patterns. The other alternative approach to $\mathrm{CNN}$ for medical images analysis and classification is application of fuzzy neural networks (FNN), in particular FNN NEFClass. FNN NEFClass was firstly suggested by D. Nauck and W. Kruse in the studies [7, 8]. It was modified and developed in the study [9] (so-called FNN NEFClass M) The main advantages of FNN NEFClass as classifier are: possibility to work with incomplete and fuzzy input data; performing fuzzy classification of input patterns (images); speed and high accuracy [7-9]. FNN NEFCLASS was firstly applied for medical images diagnostics in the problem of breast tumors classification in two classes: benign or malicious [7, 9]. The important sphere of medical information systems is pattern recognition of tumors on human organs tissue and early detection of possible cancer The important sphere of medical information systems is pattern recognition of tumors on human organs tissue and early detection of possible cancer It enables to take urgent healing and escape lethal outcome. One of such tasks is cervix epithelium state analysis and diagnostics using optical images obtained with colposcope (a method of survey of a mucous membrane of part of a neck of a uterus in the conditions of additional lighting and optical increase with the help of a colposcope). As a result of carrying out a colposcopy by the doctor the increased pictures of images with preliminary splitting into classes of diseases are provided. The problem of classification cervix epithelium state using images obtained with colposcope was considered in the studies $[10,11]$ where for its solution was suggested the application of crisp neural networks Back propagation, neural networks with radial basis functions (RBFNN) and cascade RBFNN and their efficiency investigated. The goal of this paper is the investigation of modified fuzzy neural network NEFClass $M$ for recognition of state of cervix epithelium in medical diagnostics and comparison of its efficiency with conventional RBF and cascade neural networks.

\section{Problem Statement FNN NEFClass M}

\subsection{Architecture and Training}

The problem consists in classification of obtained medical images using special medical tools: computer tomography, magneto-resonance tomography, colposcope etc.

In medical images values of the color model RGB represent components of input vector and based on this information it's needed to define, which class it should be referred to. The classifier thus refers object to one of classes according to a certain splitting of $\mathrm{N}$-dimensional space which is called as input space, and dimension of this space is a number of vector components.

For the solution of cervix epithelium state analysis and diagnostics problem using optical images fuzzy neural network (FNN) NEFClass with Gaussian membership function was suggested.

The application of FNN NEFClass is rational by following its properties:

a) NEFCLass may work with fuzzy and qualitative input information; it enables to attain better classification accuracy than conventional non-fuzzy classifiers;

b) it has accelerated convergence as compared with crisp classification methods.

The system NEFClass has 3-layer successive architecture (see figure 1). The first layer $U_{1}$ contains inputs neurons which inputs patterns are fed in. Activating of these neurons does not change usually input values. The hidden layer $U_{2}$ contains fuzzy rules, and the third layer $U_{3}$ consists of output neurons (classifiers). Activations of rule neurons and neurons of output layer with the pattern of $p$ are calculated so:

$$
\begin{array}{r}
a_{R}^{(p)}=\min _{x \in U_{1}}\left\{W(x, R)\left(a_{x}^{(p)}\right)\right\} \\
a_{C}^{(p)}=\sum_{R \in U_{2}} W(c, R) \cdot a_{R}^{(p)}
\end{array}
$$

or alternatively

$$
a_{C}^{(p)}=\max _{R \in U_{2}}\left\{a_{R}^{(p)}\right\}
$$

where $W(x, r)$ is a fuzzy weight of connection of input neuron $x$ with a rule neuron $R$, and $W(R, c)$-fuzzy weight of connection of a rule neuron $R$ with the neuron $C$ of output layer. Instead of application of operations of maximum and minimum it is possible to use other functions of so-called "t-norm" and "t- co-norm" accordingly [9].

A rule base is approximation of unknown function and describes a classification $\operatorname{task} \varphi(x)$, that $c_{i}=1, c_{i}=0$ $(j=1 \ldots m, \forall j \neq i)$,if pattern $x$ belongs to the class of $C_{i}$. 


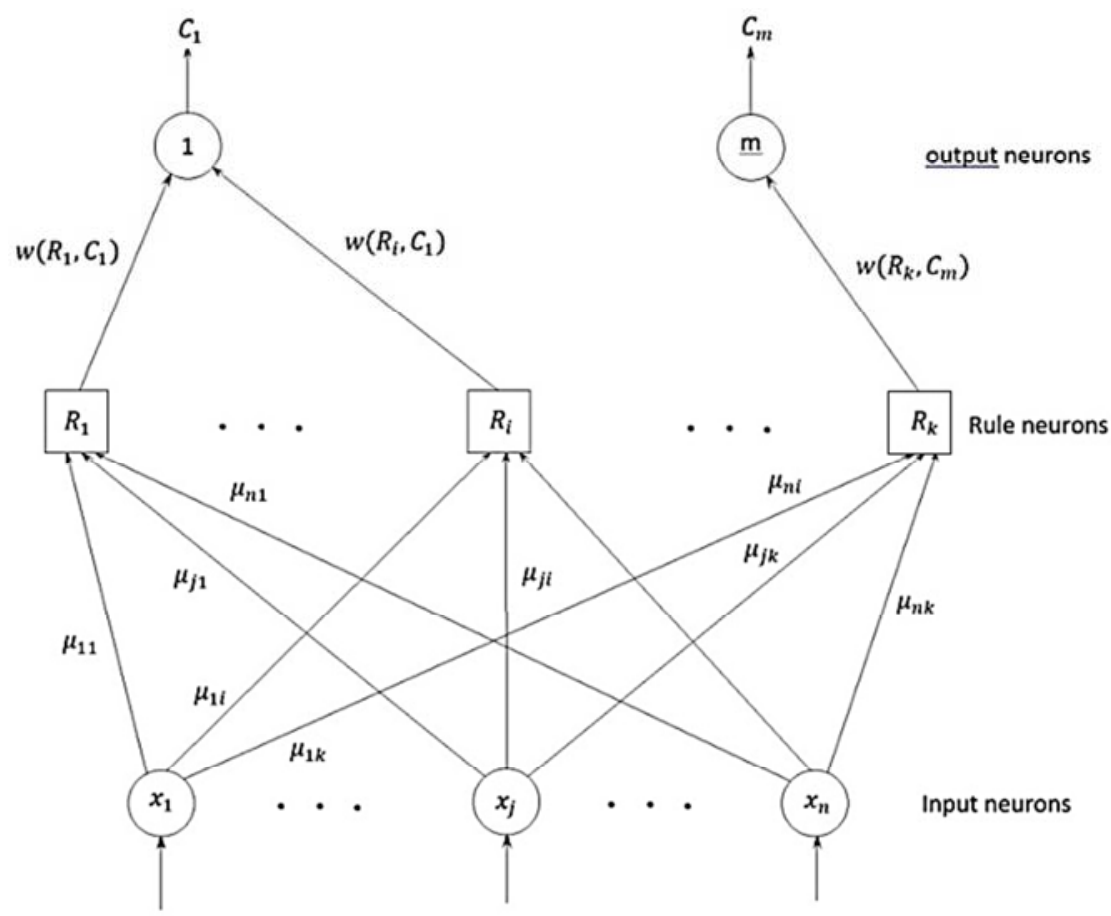

Figure 1. Structure of FNN NEFCLASS.

Every fuzzy set is marked a linguistic term, such as «large», «small», «middle» et cetera. Fuzzy sets and linguistic rules present approximation of classifying function and determine the result of the system NEFClass. They are obtained from a sample by learning. It's necessary, that for every linguistic value (for example, $\left\langle\mathrm{x}_{1}\right.$ is positive and large») there should be only one presentation of fuzzy set.

\subsection{Learning in the System NEFClass}

The system NEFClass can be built on partial knowledge about patterns. An user must define the amount of initial fuzzy sets for each of object features (number of terms) and set the value $k_{\max }$ that is a maximal number of rule nodes, which can be created in the hidden layer. For learning triangular MF are used.

Consider the system of NEFClass with $\mathrm{n}$ input neurons $x_{1}, \ldots x_{n}, k\left(k \leq k_{\max }\right)$ rule neurons and $m$ output neurons $c_{1}, \ldots c_{m}$. The learning sample of patterns is also given: $L=\left\{\left(p_{1}, t_{1}\right), \ldots\left(p_{s}, p_{s}\right)\right\}$, each of which consists of input pattern $p \in R^{n}$ and desired pattern $t \in\{0,1\}^{m}$.

A learning algorithm consists of two stages. At the first stage rule base generation is performed. On the second stage learning of parameters of membership functions (MF) of fuzzy sets is performed A learning algorithm with teacher of the system NEFClass must adapt MF of fuzzy sets. At FNN NEFCLass M Gaussian membership functions are used and for training their parameters a stochastic gradient descent algorithm and conjugate gradient algorithm with accelerated convergence were used $[12,13]$.

\subsection{Stochastic Gradient Descent Learning Algorithm}

For the first stage of the algorithm - learning rule base the first phase of the basic algorithm NEFClass is used. The second stage uses a gradient algorithm for training the feedforward neural network, which is described below [12].

Let the criterion of training fuzzy neural network, which has 3 layers (one hidden layer), be as follows:

$$
e(W)=\sum_{i=1}^{M}\left(t_{i}-N E T_{i}(W)\right)^{2} \rightarrow \min
$$

where $t_{i}$ - the desired value of the i-th output of neural network;

$N E T_{i}(W)$-the actual value of the i-th neural network output for the weight matrix $W=\left[W^{\mathrm{I}}, W^{0}\right], W^{I}=W(x, R)=$ $\mu_{j}(x), W^{O}=W(R, C)$.

Let activation function for the hidden layer neurons (neurons rules) be such:

$$
O_{R}=\prod_{i=1}^{N} \mu_{j_{i}}^{(i)}\left(x_{i}\right), j=1, k
$$

where $\mu_{j i}\left(x_{i}\right)$ membership function, which has the form (Gaussian):

$$
\mu_{j_{i}}^{(i)}(x)=e^{-\frac{\left(x-a_{j i)}\right)^{2}}{b_{j i}^{2}}}
$$

and the activation function of neurons in the output layer (weighted sum):

$$
O_{C}=\frac{\sum_{R \in U_{2}} W(R, C) \cdot O_{R}}{\sum_{R \in U_{2}} W(R, C)}
$$

or maximum function:

$$
O_{c}=\max W(R, C) \cdot O_{R} .
$$


Consider the gradient learning algorithm of fuzzy perceptron.

1. Let $W(n)$-be the current value of the weights matrix. The algorithm has the following form:

$$
W(n+1)=W(n)-\gamma_{n+1} \nabla_{w} e(W(n))
$$

where $\gamma_{n}$-the step size at $n$th iteration;

$\nabla_{w} e(W(n))$-gradient (direction), which reduces the criterion (4).

2. At each iteration, we first train (adjust) the input weight $W$, which depend on the parameters $a$ and $b$ (see the expression 6)

$$
\begin{aligned}
& a_{j i}(n+1)=a_{j i}(n)-\gamma_{n+1} \frac{\partial e(W)}{\partial a_{j i}} \\
& b_{j i}(n+1)=b_{j i}(n)-\gamma_{n+1}^{\prime} \frac{\partial e(W)}{\partial b_{j i}}
\end{aligned}
$$

where $\gamma_{n+1}^{\prime}$ - step size for parameter $b$.

$$
\begin{aligned}
& \left.\frac{\partial e(W)}{\partial a_{j i}}=-2 \sum_{k=1}^{M}\left(\left(t_{k}-N E T_{k}\right)\right) \cdot W(R, C)\right) \cdot O_{R} \cdot \frac{\left(x-a_{j i}\right)}{b_{j i}^{2}} \\
& \left.\frac{\partial e(W)}{\partial b_{j i}}=-2 \sum_{k=1}^{M}\left(\left(t_{k}-N E T_{k}\right)\right) \cdot W(R, C)\right) \cdot O_{R} \cdot \frac{\left(x-a_{j i}\right)}{b_{j i}^{3}}
\end{aligned}
$$

3. We find (train) output weight:

$$
\begin{aligned}
& \frac{\partial e\left(W^{O}\right)}{\partial W\left(R, C_{k}\right)}=-\left(t_{k}-N E T_{k}\left(W^{O}\right)\right) \cdot O_{R} \\
& W_{k}^{o}(n+1)=W_{k}^{o}(n)-\gamma^{\prime \prime}{ }_{n+1} \frac{\partial e\left(W^{O}\right)}{\partial W\left(R, C_{k}\right)}
\end{aligned}
$$

4. $n:=n+1$ and go to the next iteration.

The gradient method is the first proposed learning algorithm, it is easy to implement, but has the disadvantages:

1. converges slowly; finds a local extremum.

Therefore for training speed acceleration conjugate gradient algorithm was also implemented [13].

\section{Data Set Description}

Initial experimental data were obtained using colposcope from 185 patients in a hospital and represented RGB images of uterus. In the figure 2. some images of cervix uterus epithelium are presented.

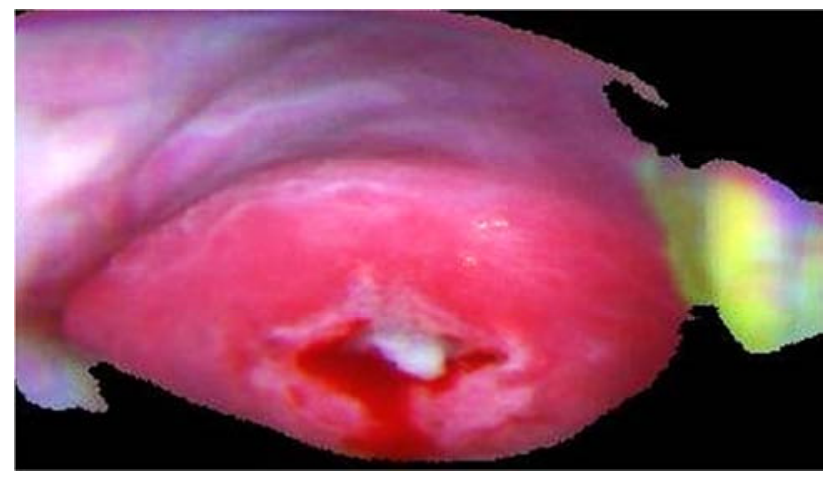

Figure 2. Image of cervix with dysplasia.

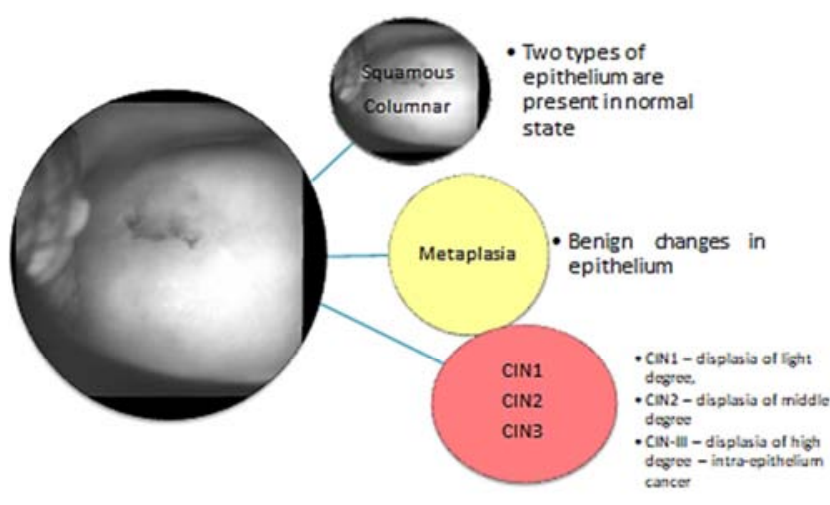

Figure 3. States of opithelium.
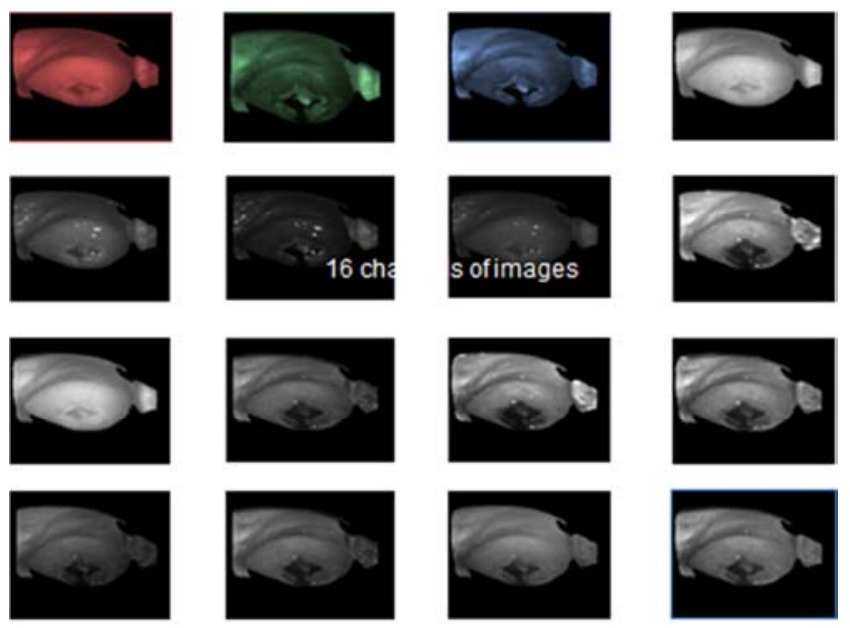

Figure 4. Uterus images in 16 channels.

The problem is to classify epithelium states in one of six classes: 1) normal state-columnar epithelium; 2) squamous epithelium (normal state); 3) metaplasia-benign changes of cervix uterus epithelium; 4) CIN1-displasia of light degree, 5) CIN 2-displasia of middle degree, 6) CIN 3-displasia of high degree- intra-epithelium cancer (see figure 3 ).

nitial images were processed by 16 channels:

a) Four ranges of reflected polarized light with polarize parallel to light source;

b) Four ranges of reflected polarized light with polarize orthogonal to light source;

c) 8 ranges of fluorescence with wave length $365 \mathrm{~nm}$ (two filter sets) (see figure 4)

\section{Experimental Investigations}

Process of experimental investigations consisted of several stages:

Preliminary processing of obtained images filtration;

Reduction of initial feature set and selection of informative features;

Segmentation as algorithms of segmentation were used Cmeans, fuzzy C-means and Kohonen feature maps [13];

Choice of Region of Interest (ROI) in the image;

Analysis and classification of epithelium state in ROI and determination of places where biopsy should be taken; 
Estimation of classification accuracy using biopsy results.

All the images was divided into training and test subsamples in given ratio (usually 70/30 \%) .

As it were used 16 input channels and ROI size was 20x20 pixels the initial set of features consisted of 6400 primary features, that is too much. Therefore, the reduction of features number was performed and for this Principal components method was applied [14]. Using PCM the most informative 14 features were selected which include $85 \%$ of initial covariance. All the experiments were performed using this set of selected features.

At the first stage the FNN NEF Class was trained using algorithms: gradient descent and conjugate gradient. After training at the second stage classification experiments at images of uterus at test subsample were performed and accuracy was estimated.

The accuracy of classification of FNN NEFClass was compared with another neural networks (NN): Back propagation $\mathrm{NN}$, Radial-basis functions $\mathrm{NN}$ and cascade RBFNN [10]. As a criterion of correct classification MAPE (\%) was used. The results of experiments for different classifiers are presented in the table 1.

Table 1. Classification accuracy of uterus epithelium state for different networks.

\begin{tabular}{lllll}
\hline Criterion & $\begin{array}{l}\text { NEFC } \\
\text { lass }\end{array}$ & $\begin{array}{l}\text { NN Back } \\
\text { propagation }\end{array}$ & RBFNN & $\begin{array}{l}\text { Cascade } \\
\text { RBFN }\end{array}$ \\
\hline False positive, \% & 0.1050 & 0.1650 & 0.1350 & 0.1325 \\
False negative, $\%$ & 0.05 & 0.0575 & 0.0250 & 0.0100 \\
Correct, \% & 0.8900 & 0.7775 & 0.8400 & 0.8575 \\
\hline
\end{tabular}

As it follows from the presented results FNN NEFClass has the best accuracy as compared with alternative classifiers. This experiment was performed in the case of 6 different classes of states.

In the next experiments four classes of states were used as classes CIN1, CIN2 and CIN3 were united in one class of cancer states. The corresponding results and classification accuracy for different classifiers are presented in the table 2.

Table 2. Classification accuracy for FNN NEFClass and RBFN with 4 classes.

\begin{tabular}{lll}
\hline Network type & $\begin{array}{l}\text { Correct } \\
\text { classification, \% }\end{array}$ & $\begin{array}{l}\text { Misclassificat } \\
\text { ion, \% }\end{array}$ \\
\hline Cascade RBF NN & 85.75 & 14.25 \\
$\begin{array}{l}\text { FNN NEF Class, best rules, } \\
\text { Gaussian MF }\end{array}$ & 90.00 & 10.00 \\
$\begin{array}{l}\text { FNN NEF Class, all rules, } \\
\text { Gaussian MF }\end{array}$ & 85.10 & 14.90 \\
$\begin{array}{l}\text { FNN NEF Class, all rules, } \\
\text { triangular MF }\end{array}$ & 80.00 & 20.00 \\
\hline
\end{tabular}

From the table 2 follows that FNN NEFClass with Gaussian MF and the best rules has the highest accuracy as compared with alternative $\mathrm{NN}$.

\section{Conclusion}

The problem of medical images of uterus epithelium analysis and recognition is considered. The problem was to classify state of uterus epithelium into one of six classes: 1) normal stat -columnar epithelium; 2) squamous epithelium (normal state); 3) metaplasia - benign changes of cervix uterus epithelium; 4) CIN1 - displasia of light degree, 5) CIN 2-displasia of middle degree, 6) CIN 3-displasia of high degree- intra-epithelium cancer

For its solution FNN NEFClass is suggested.

The experimental investigation of application FNN

NEFClass for medical images recognition on real data were carried out and classification accuracy was estimated.

The comparison of FNN NEFClass with alternative networks Back propagation, RBFNN and cascade RBFNN was performed.

The experiments confirmed the efficiency of FNN NEFClass for medical images classification in the problems of medical diagnostics.

\section{References}

[1] Doyle, S. Agner, A. Madabhushi, M. Feldman, and J. Tomaszewski, "Automated grading of breast cancer histopathology using spectral clustering with textural and architectural image features," in Proceedings of the 5th IEEE International Symposium on Biomedical Imaging (ISBI): From Nano to Macro, vol. 61. IEEE, May 2008, pp. 496-499.

[2] Y. Le Cun, Y. Bengio, and G. Hinton, "Deep learning," Nature, vol. 521, pp. 436-444, 2015.

[3] Krizhevsky, I. Sutskever, and G. E. Hinton, "Imagenet classification with deep convolutional neural networks," in Advances in NeuralInformation Processing Systems 25, 2012, pp. 1097-1105.

[4] Jianpeng Zhang, Yong Xia, Qi Wu, Yutong Xie. Classification of Medical Images and Illustrations in the Biomedical Literature Using Synergic Deep Learning, Xiv:1706.09092v1 [cs. CV] 28 Jun 2017.

[5] Nilanjan Dey. Classification Technologies Techniques for Medical ImageAnalysis and Computer-aided Diagnosis, Volume 14, 1st Edition.-Academic Press, 201.-218 p.

[6] Yin, Xiao-Xia, Hadjiloucas, Sillas, Zhang, Yanchun. Pattern Classification of Medical Images: Computer Aided Diagnosis Springer International. Springer International Publishing. 2017.-218 p. DOI. 10.1007/978-3-319-57027-3.Hardcover ISBN.978-3-319-57026-6.

[7] K. Malyshevska. The analysis of neural networks' performance for medical image classification/K. Malyshevska//International Journal "Information Content and Processing", Volume 1, Number 2, 2014.-C. 194-199.

[8] K. Malyshevska. Analysis of neural networks application for diagnostics of uterus cancer using multispectral images. System research and information technologies.-2010-№2-pp. 64-71. (rus)

[9] Detlef Nauck and Rudolf Kruse. Generating classification rules with the neuro-fuzzy system NEFCLASS. In Proc. Biennial Conf. of the Norght American Fuzzy Information Processing Society (NAFIPS'96), Berkeley, 1996.

[10] Detlef Nauck and Rudolf Kruse. New learning strategies for NEFCLASS. In Proc. Seventh International Fuzzy Systems Association World Congress IFSA'97, Vol. IV, pp. 50-55, Academia Prague, 1997. 
[11] Zaychenko Yu. P., Sevaee Fatma, Matsak A. V. Fuzzy neural networks for economic data classification//Vestnik of National Technical University of Ukraine "KPI", section "Informatic, control and c omputer engineering. Vol. 42.-2004.-pp. 121-133. (rus).

[12] Zaychenko Yu. P., Petrosyuk I. M., Jaroshenko M. S. The investigations of fuzzy neural networks in the problems of electro-optical images recognition//System research and information technologies.-2009.-№4.-pp. 61-76. (rus).
[13] M. Zgurovsky, Yu. Zaychenko. The Fundamentals of Computational Intelligence: System Approach. Springer International Publishing AG, Switzerland.-2016-308p.

[14] N. Jindal. Enhanced Face Recognition Algorithm using PCA with Artificial Neural Networks./N Jindal, V Kumar//International Journal of Advanced Research in Computer Science and Software Engineering-2013-vol 3 pp. 864-872. 